\title{
Análisis de los factores determinantes del resultado exportador en las empresas españolas del sector calzado
}

\section{Analysis of the determinants of export performance in the spanish companies from the footwear industry}

Fernando González-Ferriz

fernando.gonzalez.ferriz@ui1.es (1) 0000-0002-2007-4862

Universidad Isabel I. Calle de Fernán González, 76. 09003 Burgos, España

\section{INFO ARTÍCULO}

Recibido: 22-04-2021

Revisado: 12-06-2021

Aceptado: 23-06-2021

\section{PALABRAS CLAVE}

Internacionalización

Marketing internacional

Resultado exportador

Exportación

Gestión empresarial

Sector calzado

\section{KEYWORDS}

Internationalization

International marketing

Export performance

Exports

Management

Footwear industry

\begin{abstract}
RESUMEN
Esta investigación pretende profundizar en el comportamiento de las empresas exportadoras del sector calzado, uno de los más importantes en el levante español, que genera valor añadido a través de la moda. El testimonio de los directivos de una muestra de empresas representativas, mostrará las fortalezas y debilidades de sus procesos de internacionalización, prestando especial importancia a variables como la estrategia de marketing internacional o la propia función directiva. Por otra parte, el análisis descriptivo de los principales condicionantes del resultado exportador, mostrará que los directivos del sector están bien formados, que el entorno y las características de la empresa van a condicionar la estrategia de marketing y que ésta incorpora elementos relacionales y ciertos aspectos de las nuevas tecnologías. Todo ello repercute en la evolución hacia un entorno basado en los servicios y en el estudio del mercado con el fin de identificar de la mejor forma posible las necesidades del cliente. Finalmente, desde el punto de vista académico, se analizará la contribución de teorías como el enfoque de los recursos, las capacidades dinámicas o la teoría de la contingencia.
\end{abstract}

\begin{abstract}
This investigation pretends to examine the behaviour of the exporting companies in the footwear sector, one of the most important in the eastern region of Spain, which generates sustainable added value through fashion. The testimony of the managers from a representative selection of companies will show the strengths and weaknesses of their internationalization processes, paying a special attention to variables like the international marketing strategy or the managerial function. On the other hand, the descriptive analysis of the most important determinants of export performance in the sector, will show that: (1) managers are well trained, (2) both the environment and firm characteristics will influence the marketing strategy, and (3) marketing policies incorporate relational elements and certain aspects of new technologies. This results in the evolution towards an environment based on services, where the study of market conditions will be decisive to identify customer needs in the best possible way. Finally, the contribution of theories like the Resourced Based View (RBV), the dynamic capabilities or the contingency theory will be crucial from the academic point of view.
\end{abstract}




\section{INTRODUCCIÓN}

La globalización de las economías y el incremento de las exportaciones han sido tradicionalmente materias que han suscitado un gran interés a nivel académico. En el caso español, las ventas al exterior han supuesto una de las principales fuentes de ingresos para el país, junto a sectores como la construcción y el turismo. De hecho, si analizamos las cifras de exportación en nuestro país desde los primeros años de este siglo, observamos que han mostrado una clara y constante tendencia al alza, a excepción de los años posteriores a la crisis de 2008 y, más recientemente, a partir de 2020, con la crisis de la COVID-19 (Base de datos ESTACOM, ICEX; 2021), períodos en los que las cifras de ventas exteriores se han resentido. Si analizamos los sectores con mayor peso dentro del comercio exterior español, observamos cómo destacan entre otros la industria agroalimentaria, la automoción, los productos químicos y aquellos relacionados con la moda, jugando un papel determinante dentro de este último grupo el sector del calzado.

Cabe destacar que el sector de la moda genera una contribución cercana al 3\% del PIB español, lo cual supone más de un $4 \%$ en términos de empleo. Por su parte, el calzado ha sido el único subsector que logró recuperar los niveles pre-crisis en número de empleados con anterioridad a la era COVID (CESCE, Informe sectorial de la economía española, 2018, 2019). Con un total de 4.636 fabricantes, de los cuales más de 2.500 se encuentran en la Comunidad Valenciana (fundamentalmente provincia de Alicante), el sector calzado, netamente exportador, avanza hacia la diversificación de los mercados exteriores, con incrementos superiores al $5 \%$ en las ventas extracomunitarias, a pesar de que casi el $80 \%$ de las ventas se concentran todavía dentro de la Unión Europea (IVACE Internacional, Análisis del sector calzado, 2019). A pesar de ello, ciertas amenazas se ciernen sobre una industria que todavía no ha conseguido recuperarse de las consecuencias de la pandemia global y que mira con incertidumbre hacia el rearme arancelario en el continente norteamericano.

La importancia de la industria y su elevado peso relativo en la economía regional del levante español, nos ha llevado a un análisis pormenorizado de la estructura de sus empresas y de los factores que condicionan el resultado exportador de las mismas, así como al estudio de las características de la gestión empresarial y del perfil de sus directivos, que son, en definitiva, los máximos responsables a la hora de implantar de forma eficaz la estrategia de marketing internacional. Variables como el análisis del entorno, la calidad y compromiso de la gestión empresarial, la planificación de las políticas de marketing o la incorporación de las nuevas tecnologías al mundo empresarial, van a ser también determinantes para entender los procesos de internacionalización del sector.

La presente investigación parte de un análisis del marco teórico que presentará las principales teorías en el campo de la internacionalización. Éstas serán fundamentales para determinar las escalas del posterior análisis cuantitativo de los datos proporcionados por los directivos de una selección de empresas exportadoras en el sector del calzado español. El objetivo final será determinar los factores que van a influir sobre el resultado final de la muestra representativa de las empresas analizadas y destacar, de esta forma las fortalezas y debilidades del sector.

Tomaremos como punto de partida el análisis del estado de la cuestión, desde dos puntos de vista fundamentales: la teoría de la internacionalización por un lado y, por otro, el análisis de las principales corrientes de marketing y su evolución hacia un enfoque basado en la prestación de servicios. En este contexto serán fundamentales las aportaciones de la teoría de los recursos (tanto desde la perspectiva de los activos de la empresa como del conocimiento de sus directivos), que resultarán claves a la hora de crear valor y determinar la estrategia empresarial. Del mismo modo, el enfoque de las capacidades dinámicas contribuirá al análisis aportando la necesidad de adaptación, por parte de las empresas, a un entorno altamente cambiante. De esta forma, los directivos serán responsables de la estrategia de marketing en todo momento, introduciendo de forma continua mejoras en la gestión empresarial que permitan aumentar el beneficio.

En cuanto al tratamiento de los datos, un detallado análisis descriptivo de los datos aportados por un cuestionario estructurado en torno a los principales constructos de la investigación, aportarán la información necesaria para describir el comportamiento de las empresas en el sector considerado.

Por último, el presente estudio pretende contribuir al conocimiento científico mediante el análisis empírico de un sector tradicional de gran importancia en el tejido empresarial nacional y regional. Y lo hace 
definiendo las escalas e ítems de los constructos analizados en base a la literatura desarrollada en dos campos de gran importancia, como son la internacionalización y el marketing. Así pues, partiendo de escalas generalmente aceptadas, se ha conseguido adaptarlas a un sector específico y contribuir, de esta forma, a determinar los puntos fuertes y débiles del mismo, con el fin de aportar ciertos aspectos de mejora a la gestión empresarial de las pymes que componen el ecosistema. Se pretende, por tanto, vincular enfoques académicos contrastados a la gestión empresarial de un conjunto de empresas con unas características muy particulares.

\section{MARCO TEÓRICO}

La teoría sobre la internacionalización ha evolucionado desde un enfoque mucho más genérico que analizaba cuestiones basadas en aspectos macroeconómicos o en el estudio de las grandes corporaciones multinacionales y sus decisiones de deslocalización e implantación en el exterior, hasta un enfoque mucho más concreto, centrado en los factores que determinan el mayor o menor grado de éxito en la proyección internacional de las pequeñas y medianas empresas. Enumerar las variables que influyen en el resultado de las empresas exportadoras y cómo se relacionan entre sí ha sido el objetivo de la literatura en este campo del conocimiento en los últimos 50 años (Aaby \& Slater, 1989; Sousa et al., 2008; Chugan \& Singh, 2014; Chen et al., 2016).

El análisis empírico de la materia ha ido evolucionando según lo hacían los planteamientos teóricos, por lo que, finalmente, el problema se ha podido enfocar desde distintos puntos de vista, destacando corrientes que se han centrado en el análisis de los recursos de la empresa, del conocimiento, del entorno institucional, o de las capacidades dinámicas. Todos ellos apuntan en dos direcciones: el análisis de los activos tangibles, y sobre todo intangibles de la empresa, y el efecto de la continua evolución del entorno sobre los mismos.

Los modelos gradualistas de la década de los 70, impulsados por investigadores de la escuela nórdica como Johanson y Vahlne, determinaban que las empresas seguían, en la mayoría de los casos, un proceso gradual de internacionalización. Solo una vez que hayan adquirido la suficiente experiencia en los mercados nacionales, serán capaces de emprender la aventura internacional. Un proceso de aprendizaje, por tanto, les permitiría abordar primero los mercados con una menor "distancia psíquica" y características similares al mercado de origen, antes de centrarse en mercados de mayor dificultad. Cuestiones como la innovación o la gestión del conocimiento también han sido determinantes para muchos seguidores de esta corriente.

Los enfoques relacionales que se centran en el desarrollo de redes, son también fundamentales en la evolución del sustrato teórico en los procesos de internacionalización. Así pues, autores como Morgan y Hunt (1994), Gummesson (2002) o Payne y Frow $(2005,2008,2017)$ determinaron las bases para el desarrollo de relaciones a largo plazo basadas en la confianza y el compromiso, no solo con clientes, sino también con proveedores y otros miembros clave en el proceso de comercialización. Estas relaciones, además, toman la forma de redes cuando perduran en el tiempo y son la base para un nuevo concepto marketing basado más en los servicios y la atención al cliente que en el propio producto en sí (Vargo \& Lusch, 2004, 2016). Cuestiones como la orientación al mercado y el posicionamiento del cliente en el centro de la estrategia de marketing cobran ahora una especial importancia, sobre todo en lo que a estrategia de marketing internacional se refiere. Se pretende fidelizar al cliente y establecer relaciones a largo plazo con él, haciéndole sentir parte de la empresa.

El enfoque basado en los recursos (RBV) es quizás el de mayor repercusión en lo que a teoría de la internacionalización se refiere (Wernerfelt, 1984, 1995; Prahalad \& Hamel, 1990; Barney, 1991; Nelson, 1991; Teece et al., 1997; Katsikeas et al., 2000). Considerada tanto desde el punto de vista de la gestión empresarial como del marketing, se centra en el estudio de las capacidades de la empresa (activos tangibles e intangibles) que cada empresa logra desarrollar con el fin de conseguir una ventaja competitiva que solo ella posea y que le diferencie de la competencia. Algunos de estos activos son la relación con el cliente, el reconocimiento de la marca, o la generación de tecnología y conocimiento propios. 
La teoría de los recursos siguió evolucionando hasta la teoría de las capacidades dinámicas para incorporar un aspecto fundamental como es el cambio constante al que se encuentra sometido el entorno, aportando dinamismo al modelo. Investigadores como Desarbo et al. (2005), Song et al. (2008), Murray et al. (2011), o Parnell et al. (2015) defienden que esos cambios en el entorno deben ser interpretados por los directivos de la empresa porque afectan a la estrategia empresarial. Cobra, por tanto, una especial importancia el papel del conocimiento y la gestión empresarial, hasta el punto de que muchos autores lo consideran como el activo intangible más importante. De forma paralela a este enfoque, la teoría de la contingencia determina que una estrategia puede ser válida para una empresa en un momento determinado y no serlo para otra empresa u otro momento en el tiempo, debido a esos cambios imprevisibles (Chen et al., 2016).

Por último, desde el punto de vista del marketing, cabe destacar la importancia de nuevos enfoques que incorporan aspectos tan importantes como el marketing digital, o el análisis del big data en las empresas, concepto este último analizado por Kotler $(2010)$ y Kotler et al. $(2016,2021)$ cuando describen la evolución del marketing 1.0 hasta una última versión 5.0. Las diferentes etapas coinciden, además, con cambios importantes en las características de los consumidores, dando lugar a formas de actuar distintas según las necesidades de los mismos. Éstos y otros autores destacan la necesidad de considerar las estrategias de marketing desde una perspectiva holística en la que se van a incorporar aspectos fundamentales como la relación con clientes o con cualquier agente que contribuya de forma importante a la cadena de valor de la empresa. Será fundamental también en este proceso la incorporación de las nuevas tecnologías, en particular Internet, a los procesos de marketing, aspecto que será analizado desde el punto de vista de la teoría de los recursos (Resource Based View) y que ha sido analizado de forma exhaustiva por autores como Song et al. (2008) o Prasad et al. (2001).

Otra cuestión a destacar en el proceso de evolución mencionado en el párrafo anterior, es el cambio en la concepción del marketing, lo cual supone una transición desde una disciplina basada en el análisis del producto, hasta otra que otorga especial atención a la satisfacción de las necesidades del consumidor y a la fidelización del cliente (Vargo \& Lusch, 2004; 2016). Esto no quiere decir que se rompa con las aportaciones tradicionales, sino más bien que el marketing actual se basa en el tradicional. Valga como ejemplo el uso de las políticas operativas de marketing mix (producto, precio, promoción y distribución) que siguen utilizándose hoy en día, a pesar de haber sido concebidas hace más de cinco décadas.

\subsection{Factores determinantes en el proceso de internacionalización}

Como ya se ha comentado, el análisis empírico en el campo de la internacionalización se ha centrado en las últimas décadas en un análisis microeconómico que toma como unidad básica de análisis la empresa exportadora, siendo éstas pequeñas y medianas empresas en la mayoría de los casos.

Los primeros estudios, que datan de la década de los 70 (Bilkey, 1978; Aaby \& Slater, 1989) se centraban en un análisis interno y obviaban una serie de factores externos provenientes del entorno, que serían incorporados en años posteriores (Cavusgil \& Zou, 1994; Zou \& Stan, 1998). Por otra parte, la tendencia natural de la literatura derivó hacia la consideración de la estrategia de marketing internacional como uno de los factores más importantes en el proceso de internacionalización, representando el papel de variable intermedia ya que, por una parte dependía de factores como el entorno, las características de la empresa y los directivos, y por otra, sería fundamental para determinar el resultado final de la compañía (Katsikeas et al., 2000, 2006; Leonidou et al., 2002; Chen et al., 2016; Chabowski et al., 2018). Esta variable ha sido analizada desde múltiples puntos de vista, y a ella se han ido incorporando nuevos aspectos como el marketing relacional o el uso de las nuevas tecnologías.

Por último, cabe destacar la importancia de los trabajos de Chugan y Singh (2014) y de Chen et al. (2016) ya que van a contribuir a la determinación del conjunto de factores determinantes en el proceso de internacionalización (ver tabla 1), como punto de partida de este trabajo. 
Tabla 1. Factores determinantes en el proceso de internacionalización.

\section{VARIABLES INTERNAS}

\section{Activos tangibles e intangibles}

- Tamaño y años de actividad

- Experiencia internacional (empresa)

- Innovación tecnológica

- Recursos /capacidades

\section{Características de la gestión}

- Experiencia internacional (managers)

- Compromiso y actitud hacia la exportación

- Desarrollo de redes

Políticas de RSC (resp. social corporativa)

\section{VARIABLES EXTERNAS}

Características de los mercados internacionales

- Distancia física y psíquica

- Entorno socio-cultural

- Entorno político y legal

- Barreras de entrada

\section{Características del mercado nacional}

- Apoyo institucional a la exportación

- Entorno legal y político

- Barreras a la exportación

(controles, cuotas, normativa)

Estrategia de marketing internacional

- Planificación del marketing estratégico

- Políticas de marketing mix

- Uso de las Nuevas Tecnologías

Fuente: Elaborado por el autor, según Chugan y Singh (2014); Chen et al.(2016).

\section{METODOLOGÍA}

Una vez analizados los factores que condicionan el grado de éxito en el proceso de internacionalización, el siguiente paso dentro de nuestra investigación consiste en determinar cuáles de ellos van a describir mejor la realidad del sector de la fabricación y venta de calzado en España, uno de los más significativos en lo que a moda se refiere. En este sentido, es evidente que la estrategia de marketing internacional va a ser determinante en el análisis del resultado exportador, al tratarse de una industria que debe interpretar adecuadamente las tendencias del mercado y los gustos del consumidor para desarrollar un producto de acuerdo a esas necesidades.

Las características de los directivos y el tipo de gestión que van a llevar a cabo será otro de los factores determinantes en este estudio, ya que éstos van a ser los responsables de analizar el entorno (fundamentalmente en los mercados internacionales) con el fin de implantar las estrategias más adecuadas que permitan alcanzar un mayor beneficio.

Finalmente, esas estrategias empresariales van a depender de otros factores como las características de la propia empresa, constructo que también será analizado. Todo ello, nos lleva a la definición de una serie de hipótesis que se pretenden demostrar gracias al análisis descriptivo de los ítems considerados en esta investigación:

- $\mathrm{H}_{1}$ : La formación y habilidades de los directivos (management) en el entorno internacional presentan una relación positiva en el resultado empresarial.

- $\mathrm{H}_{2}$ : Las características del entorno internacional van a condicionar la estrategia de marketing internacional $y$, consecuentemente, el resultado final de la empresa.

- $\mathrm{H}_{3}$ : Las características de la empresa (fundamentalmente tamaño de la misma y años de experiencia en el sector) van a condicionar la estrategia de marketing internacional y afectan positivamente al resultado empresarial.

- $\quad \mathrm{H}_{4}$ : La estrategia de marketing internacional se adapta a las necesidades actuales del entorno e incorpora aspectos como el marketing relacional y el uso de nuevas tecnologías, que influyen positivamente en el rendimiento empresarial.

Para llevar a cabo el estudio, basado en el análisis estadístico descriptivo, se definió un cuestionario que fue entregado a los directivos de una muestra representativa de empresas del sector entre los años 2018 y 2019. 
La idoneidad de las empresas consideradas se basó en factores como la experiencia en el mercado, la regularidad de las ventas exteriores y el reconocimiento de marca. El dimensionamiento del sector se llevó a cabo utilizando la base de datos empresariales ORBIS (2018) que permite el filtrado por códigos NACE (Nomenclatura estadística para las actividades económicas a nivel europeo) así como distintos informes específicos de la industria (CESCE 2018, 2019; IVACE Internacional, 2019) llegando a la conclusión de que aproximadamente la mitad de las 2.500 empresas localizadas en el levante español venden sus productos en el exterior. La muestra final consistió en un total de 25 compañías, consideradas como exportadores regulares, y conformes a la tipología que de empresa que encontramos en esta industria.

El cuestionario de preguntas cerradas (ver Anexo I) recogía tanto información objetiva sobre facturación y características de la empresa, como apreciaciones de sus directivos en cuanto a comportamiento exportador y efectividad de la gestión empresarial y de sus políticas de marketing en comparación con la competencia. Para ello se utilizó una escala de Likert de 7 puntos en los ítems que lo permitían, correspondiéndose 1 con la peor y 7 con la mejor valoración. Las variables analizadas (ver figura 1) fueron: entorno en los mercados internacionales, calidad de la gestión empresarial (management), características de la empresa, estrategia de marketing (analizada desde el punto de vista estratégico, con la incorporación de aspectos clave del marketing relacional y el uso de las nuevas tecnologías) y rendimiento empresarial.
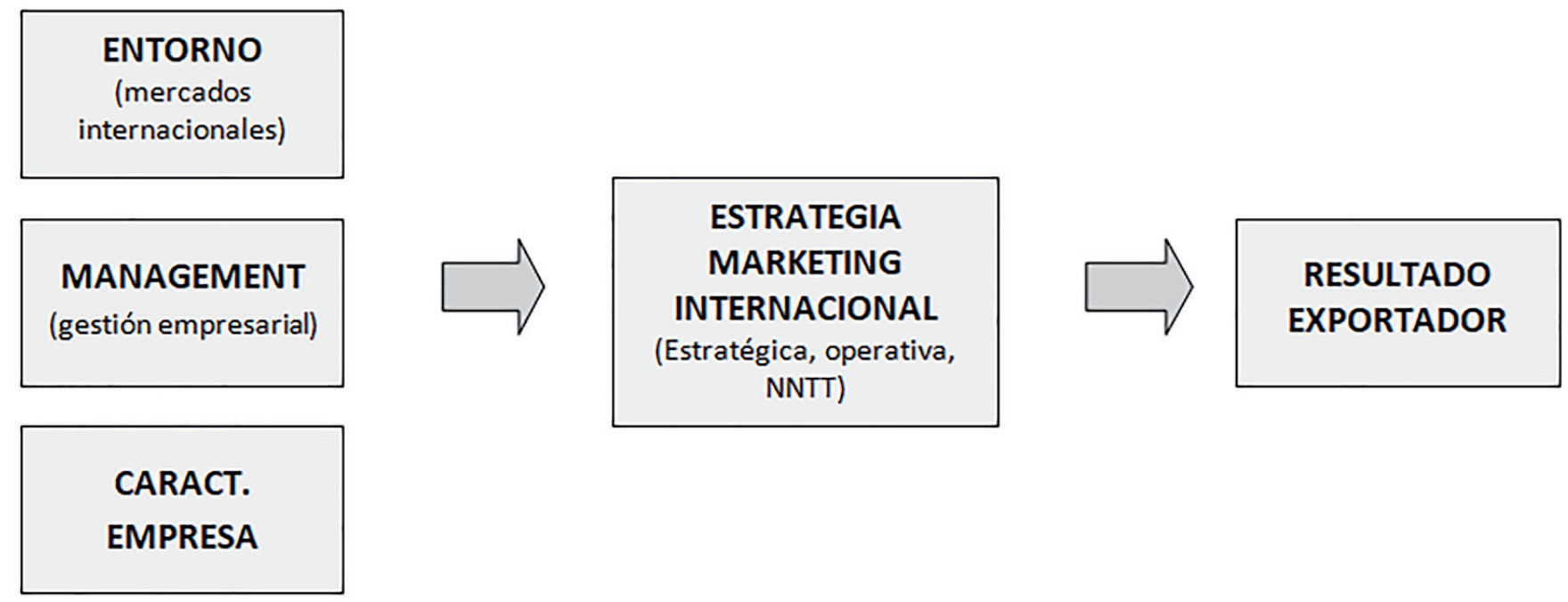

Figura 1. Variables que afectan al resultado exportador de las empresas en el sector calzado español. Fuente: Elaboración propia.

El formulario, diseñado con Google Forms, fue enviado por Internet a los directivos de las 25 empresas, siendo necesario un posterior contacto telefónico con alguno de ellos. Los resultados fueron tratados desde el punto de vista del análisis estadístico descriptivo con el software IBM SPSS 20 y las tablas dinámicas de Excel, siendo presentados en este estudio en forma de escala decimal para una mejor comprensión y análisis.

Respecto a la construcción de escalas, se consideró la siguiente literatura: (1) Desarbo et al., 2005; Song et al., 2008; Murray et al., 2011; Morgan y Katsikeas, 2012; Parnell et al., 2015, para las variables de management y marketing estratégico (que analizan el problema desde el enfoque de los recursos de la empresa y las capacidades dinámicas), (2) Leonidou et al., 2002 y Morgan et al., 2012, para las políticas de marketing operativo, (3) Prasad et al., 2001, para el uso de las nuevas tecnologías en la estrategia de marketing internacional y (4) White et al., 1998; Baldauf et al., 2000; Green et al., 2008; Alvarez, 2004; Chugan \& Singh, 2014; Chen et al., 2016 para el entorno. Por último, fue decisiva para valoración del resultado exportador de la empresa la aportación de Sousa, 2004. 


\section{RESULTADOS}

Una vez definidos el marco teórico y la metodología, procederemos a la presentación de los resultados obtenidos de la estadística descriptiva en cinco grandes bloques según el tipo de constructo analizado. Para ello mostraremos las principales contribuciones de la literatura junto a las conclusiones del sector que estamos analizando, cuestiones que nos ayudarán a determinar el grado de cumplimiento o rechazo de las hipótesis planteadas en el apartado anterior.

\subsection{Los directivos (management)}

El concepto de management ha sido ampliamente explorado por la literatura y ha ido transformándose desde una concepción mucho más económica basada en la división del trabajo y la búsqueda de la eficiencia a través de la especialización en tareas y procesos, hasta otra mucho más humana basada en teorías del comportamiento, que prestan especial atención a cuestiones como la motivación, el liderazgo, la comunicación y el trabajo en equipo.

Además, esta variable se ha analizado en multitud de investigaciones empíricas desde el punto de vista de los recursos (Resource Based View-RBV) y más concretamente del conocimiento (Knowledge Based View-KBV), otorgándole un papel fundamental a la figura del directivo en el proceso de análisis del entorno para implementar la estrategia de marketing más adecuada en cada momento y contribuir de esa forma al incremento del resultado empresarial (Barney, 1991; Grant, 1996; Ruzzier et al., 2006; Bouncken et al., 2015).

Desde el punto de vista de la internacionalización, las principales habilidades y conocimientos de los directivos que tienen un efecto probado en el beneficio de la empresa vienen recogidas por distintos autores (Lages \& Montgomery, 2005; Stöttinger \& Holzmüller, 2001; Contrator et al., 2005; Fernández \& Alegre, 2015). La tabla 2 muestra los principales ítems, valores medios y la desviación estándar de cada uno de ellos en el caso de las empresas del sector calzado analizadas en este estudio.

Así pues, se observa que los ítems con mayor valoración corresponden al alto grado de experiencia en el sector de los directivos y a los conocimientos asociados al marketing internacional, los idiomas y las nuevas tecnologías. Podemos destacar, a modo de conclusión, que los directivos del sector calzado están bien formados, si bien podrían considerarse como puntos débiles, susceptibles de mejora, las habilidades para la comunicación en la empresa y para la gestión de equipos.

Por otra parte, se observó que aquellas empresas con unos valores pormenorizados más altos en conocimientos y habilidades de sus directivos (ver tabla 7), presentaban también un mayor grado de satisfacción con el proceso de exportación y las cifras de ventas en el exterior.

El análisis descriptivo de los ítems de este constructo, nos lleva, por tanto, a aceptar como válida la primera de las hipótesis planteadas, ya que una mayor formación y orientación de los directivos hacia los mercados internacionales se asocia a valores más altos en lo que a beneficio empresarial se refiere. Esto se explica por la importancia de la función directiva en el proceso de internacionalización, tanto a la hora de promover los cambios necesarios en la organización, como a la hora de analizar las características del entorno para implantar la estrategia de marketing internacional más adecuada. Esta variable, por tanto, no opera de forma independiente, sino que será afectada por condicionantes externos (fundamentalmente provenientes de los distintos escenarios internacionales en los que opere la empresa), que son difíciles de controlar. Las características de ese entorno y la influencia que va a ejercer sobre el resto de variables se analizan con detalle en el siguiente apartado. 
Tabla 2. Ítems y valoraciones de los directivos.

\begin{tabular}{|l|c|c|}
\hline \multicolumn{1}{|c|}{ ÍTEMS MANAGEMENT } & Valores medios & Desviación estándar \\
\hline MN1: Dirección general & 7,45 & 1,14 \\
\hline MN2: Habilidades de marketing & 7,02 & 1,35 \\
\hline MN3: Conocimiento de idiomas & 7,58 & 1,33 \\
\hline $\begin{array}{l}\text { MN4: Conocimiento de Informática y } \\
\text { Nuevas Tecnologías }\end{array}$ & 7,52 & 1,48 \\
\hline MN5: Experiencia en el sector & 8,51 & 0,55 \\
\hline $\begin{array}{l}\text { MN6: Experiencia en mercados interna- } \\
\text { cionales }\end{array}$ & 7,52 & 1,19 \\
\hline MN7: Visión general de la empresa & 7,14 & 1,18 \\
\hline MN8: Orientación a la exportación & 7,45 & 1,41 \\
\hline $\begin{array}{l}\text { MN9: Habilidades para facilitar la co- } \\
\text { municación en la empresa }\end{array}$ & 6,77 & 1,26 \\
\hline MN10: Gestión de equipos & 6,77 & 1,33 \\
\hline
\end{tabular}

Fuente: Elaboración propia.

\subsection{El entorno internacional}

El entorno ha sido considerado tradicionalmente como una variable externa que no puede ser controlada por la empresa pero que va a condicionar los recursos y la estrategia de la compañía, y, consecuentemente, el resultado empresarial. A pesar de que la literatura ha analizado habitualmente esta variable desde dos vertientes (entorno nacional e internacional), nuestro estudio se centrará únicamente en la segunda ya que las características del entorno doméstico no van a ejercer una gran influencia sobre el modelo planteado.

Por tanto, en lo que respecta al entorno internacional, el concepto de distancia psíquica se une al de distancia física y determina que los países con similitudes culturales, sociales, políticas o legales son más fáciles de gestionar por las empresas exportadoras (Sousa et al., 2008; Sousa \& Lengler, 2009; Chugan \& Singh, 2014, Chen et al., 2016). El mayor o menor nivel de competencia en los mercados exteriores también juega un papel fundamental en el resultado exportador, así como la existencia de barreras y trabas a la importación (White et al., 1998; Zou \& Stan, 1998; Baldauf et al., 2000; O'Cass \& Julian, 2003; Alvarez, 2004; Lages \& Montgomery, 2005; Green et al., 2008).

En el caso del calzado español, los directivos encuestados destacan como aspectos más favorables para la apertura de mercados exteriores el bajo nivel de competencia en el sector de destino y la detección de nuevas necesidades de los consumidores, así como la estabilidad en dichos mercados (ver tabla 3). Consideran también como aspectos positivos la ausencia de barreras o trabas a la importación, y las similitudes en términos políticos y legales. Otorgan, sin embargo, menos importancia a las similitudes en el idioma o en las costumbres.

Estos resultados y su relación con los principales indicadores de eficiencia empresarial, analizados mediante el correspondiente análisis estadístico, van a ser determinantes para la confirmación de la segunda de las hipótesis planteadas, ya que las características del entorno en los mercados de destino van a condicionar la estrategia de marketing de la empresa, y por tanto, el rendimiento. Así pues, mayores similitudes en los mercados exteriores, implicarán una menor adaptación de las políticas de marketing internacional. Del mismo modo, un mayor grado de estabilidad y la ausencia de barreras y trabas a la importación, resultarán en una política de marketing más efectiva y en un mejor resultado empresarial. 
Tabla 3. Ítems y valoraciones del entorno internacional.

\begin{tabular}{|l|l|l|}
\hline \multicolumn{1}{|c|}{ ÍTEMS ENTORNO } & \multicolumn{1}{|c|}{$\begin{array}{c}\text { Valores } \\
\text { medios }\end{array}$} & $\begin{array}{c}\text { Desviación } \\
\text { estándar }\end{array}$ \\
\hline ENV1: Bajo nivel de competencia en el sector & 7,08 & 1,16 \\
\hline ENV2: Posibilidad de mantener altos márgenes & 6,89 & 1,40 \\
\hline ENV3: Detección de nuevas necesidades de los consumidores & 7,52 & 1,22 \\
\hline ENV4: Estabilidad del mercado (pocos cambios a nivel tecnológico, político, social...) & 7,08 & 1,33 \\
\hline ENV5: Existencia de barreras arancelarias & 7,02 & 1,18 \\
\hline ENV6: Existencia de barreras no arancelarias (normativa, requisitos técnicos...) & 6,46 & 1,25 \\
\hline ENV7: Similitudes en idioma/costumbres & 5,71 & 1,22 \\
\hline ENV8: Similitudes en términos políticos y legales & 6,21 & 1,17 \\
\hline ENV9: Similitud de las políticas de marketing & 6,58 & 0,77 \\
\hline ENV10: Existencia de políticas de apoyo a la exportación & 6,71 & 1,16 \\
\hline
\end{tabular}

Fuente: elaboración propia

\subsection{Características de la empresa}

Las características de la empresa han sido consideradas en muchas ocasiones desde el punto de vista de las capacidades y los recursos (Resource Based View-RBV) cuando se analiza la literatura sobre internacionalización, habiendo sido demostrada la relación positiva entre éstas y la estrategia empresarial (Chen et al., 2016; Parnell et al., 2015; Lipuma et al., 2013; Zhu et al., 2013; Sousa et al., 2008; Desarbo et al., 2005). No en vano, determinadas características pueden ser consideradas como activos intangibles, fuente de diferenciación con respecto a la competencia y generadoras de valor añadido.

Además, el análisis empírico de los factores que determinan el resultado exportador ha analizado la estructura de la empresa desde dos puntos de vista: características de la empresa y capacidades empresariales (White et al., 1998; Baldauf et al., 2000; Francis \& Collins-Dodd, 2004).

En lo que respecta a las características de la empresa, tanto el tamaño como su experiencia en mercados exteriores han resultado clave para determinar la estrategia de marketing internacional.

Cuando analizamos este aspecto en el sector del calzado (ver tabla 4), observamos que las cifras sobre facturación y número de empleados muestran una estructura de pyme en la mayoría de los casos. Se trata de un sector muy fragmentado, con empresas familiares de tamaño medio y plantillas reducidas. La dispersión con respecto a la media también es elevada, lo cual muestra que las cifras son dispares, no atendiendo a una estructura generalizada. Si analizamos el número medio de empleados, observamos que la cifra se encuentra por debajo de la centena, pudiéndose explicar este hecho por la existencia de empresas comercializadoras sin estructura productiva.

Observamos también que se trata de un sector maduro, con una media superior a los 28 años de existencia, reduciéndose la cifra hasta los casi 20 años de experiencia en exportación. En cuanto al número de países a los que exportan, la media se encuentra en torno a los 25 , resultando una cuota de exportaciones sobre el nivel total de facturación (intensidad exportadora) ligeramente superior al $60 \%$, si bien la mayor parte de ellas se realizan dentro de la Unión Europea (aunque los esfuerzos por diversificar mercados son una constante en el sector).

Por otra parte, el análisis descriptivo de los datos del cuestionario ha confirmado que, las empresas con mayor tamaño y experiencia en los mercados internacionales, exportan a un mayor número de países y realizan un mayor esfuerzo para adaptar sus estrategias de marketing internacional a los mercados exteriores, viéndose reflejado en un mayor nivel de satisfacción con el resultado final de la empresa (ver tabla 7). 
Tabla 4. Ítems y valoraciones de las características de la empresa.

\begin{tabular}{|l|c|c|}
\hline \multicolumn{1}{|c|}{ ÍtEMS CARACTERÍSTICAS DE LA EMPRESA } & Valores medios & Desviación estándar \\
\hline FC1:Facturación total (€) & 22.460 .870 & 47.488 .431 \\
\hline FC2: N. empleados & 99,74 & 217,16 \\
\hline FC3: N. años funcionamiento & 28,61 & 13,36 \\
\hline FC4: N. años exportando & 19,43 & 9,09 \\
\hline FC5: N. países a los que exporta & 24,13 & 19,80 \\
\hline FC6: Intensidad exportadora: cifra exportación/total de ventas (\%) & 60,26 & 22,12 \\
\hline FC7: Exportaciones Unión Europea / Total exportaciones (\%) & 49,09 & 20,11 \\
\hline
\end{tabular}

Fuente: Elaboración propia.

Por todo lo anterior, podemos concluir que, en base a las conclusiones del análisis estadístico de los datos, se cumple la tercera hipótesis, demostrándose la relación positiva entre las características de la empresa (fundamentalmente tamaño y experiencia en mercados exteriores) y una adecuada implantación de la estrategia de marketing internacional, teniendo como resultado un incremento del beneficio empresarial. Esta relación, como ya se ha dicho, ha sido defendida por un gran número de autores a lo largo de las últimas décadas. Si entramos en detalle observamos que, empresas con mayor tamaño pueden permitirse contratar directivos con una mayor formación y orientación al mercado, lo cual será determinante en el grado de consecución de los objetivos de la empresa. Las empresas con mayor experiencia en los mercados, también han sido capaces de desarrollar, de forma más eficiente, activos intangibles relacionados con el conocimiento de los mercados internacionales.

\subsection{La estrategia de marketing internacional}

La estrategia de marketing internacional es una de las variables más complejas, y a la vez más importantes, dentro del análisis de los factores que determinan el éxito de las empresas exportadoras. Esto se debe al hecho de que actúa como una variable intermedia sobre la que influyen factores como el entorno, las características de la empresa y la función directiva, resultando a su vez clave para la obtención un mejor o peor resultado empresarial (Katsikeas et al., 2000, 2006; Leonidou et al., 2002; Chen et al., 2016).

Este constructo ha sido tradicionalmente analizado en el mundo académico desde el punto de vista de los recursos (Resource Based View-RBV), adoptando en los últimos tiempos aspectos relacionados con la teoría de las capacidades dinámicas (dynamic capabilities) y de la contingencia (contingency theory). Así pues, la estrategia de marketing va a formar parte (al igual que las características de la empresa) del conjunto de recursos desarrollados por la empresa para determinar su ventaja competitiva y diferenciarse de la competencia. Pero no va a permanecer estática a lo largo del tiempo, ya que las circunstancias cambian constantemente y la estrategia debe adaptarse a las nuevas necesidades del entorno y de los consumidores.

Serán determinantes aspectos relacionados con las políticas de marketing estratégico (conocimiento de clientes y competencia, planificación de las actividades de marketing o habilidad para segmentar los mercados), pero también otros relacionados con las nuevas tendencias de marketing relacional (relación con los miembros del canal, cuidado de la imagen corporativa...) y de la incorporación de las nuevas tecnologías al proceso, recogiéndose estos aspectos en una amplia colección de trabajos (Desarbo et al., 2005; Song et al., 2008; Murray et al., 2011; Morgan \& Katsikeas, 2012; Parnell et al., 2015; Leonidou et al., 2002; Morgan et al., 2012; Prasad et al., 2001).

En el sector que nos ocupa (ver tabla 5), podemos observar que los ítems a los que los propios directivos otorgan mayor valoración (cuando son comparados con la competencia) son el conocimiento de clientes y competencia, el cuidado de la imagen corporativa y la efectividad de las políticas de marketing relacional 
(relación con miembros del canal, efectividad del servicio post-venta y del equipo comercial, y habilidad para retener clientes). Presentan, sin embargo, valores más modestos aspectos como la planificación de las actividades de marketing, el desarrollo de los proyectos de investigación de mercados y la incorporación de las nuevas tecnologías a las políticas de marketing.

Tabla 5. Ítems y valoraciones de la estrategia de marketing internacional.

\begin{tabular}{|c|c|c|}
\hline ÍTEMS ESTRATEGIA DE MARKETING INTERNACIONAL & $\begin{array}{l}\text { Valores } \\
\text { medios }\end{array}$ & $\begin{array}{c}\text { Desviación } \\
\text { estándar }\end{array}$ \\
\hline MS1: Conocimiento de los clientes & 7,52 & 0,90 \\
\hline MS2: Conocimiento de la competencia & 7,58 & 1,16 \\
\hline MS3: Planificación de las actividades de marketing & 6,65 & 1,34 \\
\hline MS4: Integración actividades de marketing (mismas políticas en todos los mercados) & 6,15 & 1,23 \\
\hline MS5: Habilidad para segmentar y reconocer mercados & 6,71 & 1,16 \\
\hline MS6: Desarrollo de proyectos de investigación de mercados & 6,21 & 1,37 \\
\hline MS7: Relación con los miembros del canal (agentes, distribuidores...) & 7,52 & 0,90 \\
\hline MS8: Cuidado de la imagen corporativa (políticas ambientales, éticas, sociales...) & 7,58 & 1,33 \\
\hline MS9: Habilidad para retener a los clientes & 7,45 & 0,93 \\
\hline MS10:Efectividad del servicio post-venta & 7,64 & 0,87 \\
\hline MS11: Efectividad del equipo comercial & 7,45 & 0,93 \\
\hline MS12: Incorporación de las nuevas tecnologías en las políticas de marketing & 6,89 & 1,30 \\
\hline
\end{tabular}

Fuente: Elaboración propia.

Estos resultados, por tanto, nos llevarán a la aceptación de forma parcial de la última de las hipótesis planteadas. Si bien las empresas están haciendo un gran esfuerzo para incorporar aspectos del marketing relacional a la estrategia de marketing internacional, queda todavía margen para la mejora en los procesos de incorporación de las nuevas tecnologías a esas estrategias. Estas conclusiones, por otra parte, no son de extrañar, ya que el tamaño reducido de las empresas del sector, dificulta, en gran medida, el acceso a las nuevas tecnologías (análisis de datos de ventas, desarrollo de plataformas de venta...). Finalmente, se puede afirmar que tanto las políticas relacionales de marketing como el uso de las nuevas tecnologías tienen un efecto positivo en el grado de satisfacción empresarial (ver tabla 7).

\subsection{El resultado exportador}

La última de las variables que vamos a analizar es el resultado exportador, entendiendo éste como el grado de eficiencia del proceso de internacionalización. Para ello nos basamos en el estudio de Sousa (2004) que recoge los principales indicadores para medir esta variable, distinguiendo entre aspectos objetivos y subjetivos. Debido a que las cifras de carácter objetivo pueden ser obtenidas mediante informes financieros, se ha optado por considerar indicadores subjetivos, prestando especial atención a los incluidos en la tabla 6.

Así pues, los directivos muestran una cierta satisfacción con los procesos de internacionalización (7,52 sobre 10), en especial en lo que a volumen de exportaciones se refiere. Igualmente muestran un notable grado de complacencia con la cuota de mercado en exportación y con el crecimiento de las ventas exteriores. En el lado opuesto, observamos que podría mejorar el nivel de satisfacción con el margen comercial de las exportaciones o el acceso a los mercados internacionales. 
Tabla 6. Ítems y valoraciones del resultado exportador.

\begin{tabular}{|l|l|l|}
\hline \multicolumn{1}{|c|}{ ÍTEMS RESULTADO EXPORTADOR } & $\begin{array}{c}\text { Valores } \\
\text { medios }\end{array}$ & $\begin{array}{c}\text { Desviación } \\
\text { estándar }\end{array}$ \\
\hline EP1: Volumen de exportaciones & 8,01 & 0,97 \\
\hline EP2: Crecimiento de las exportaciones & 7,45 & 1,10 \\
\hline EP3: Cuota de mercado (exportación) & 7,14 & 1,50 \\
\hline EP4: Grado de diversificación (no de países a los que exporta) & 7,02 & 1,18 \\
\hline EP5: Acceso a los mercados internacionales & 6,71 & 1,46 \\
\hline EP6: Rentabilidad de las exportaciones & 7,02 & 1,18 \\
\hline EP7: Margen comercial de las exportaciones & 6,83 & 1,18 \\
\hline EP8: Satisfacción general con la exportación & 7,52 & 1,11 \\
\hline
\end{tabular}

Fuente: Elaboración propia.

Cabe destacar que, si bien estos resultados son importantes porque nos permiten conocer el nivel de satisfacción de los directivos con los procesos de internacionalización de sus empresas, contribuyen también de una forma muy significativa a nuestra investigación, ya que permiten asociar cada uno de los constructos anteriormente analizados a variables como el nivel de satisfacción general.

Para ello, la Tabla 7 desglosa los valores medios obtenidos en aquellas respuestas que presentan un mayor nivel de satisfacción con el proceso de internacionalización (valoración de 6 o 7 en la escala original de 7 puntos) y los valores medios asociados a niveles más bajos de satisfacción (por debajo de 6).

Tabla 7. Valores medios por ítem analizado y nivel de satisfacción con el resultado exportador.

\begin{tabular}{|l|c|c|}
\hline \multicolumn{1}{|c|}{ SELECCIÓN DE ÍTEMS MÁS REPRESENTATIVOS } & \multicolumn{2}{c|}{ Valores medios } \\
\cline { 2 - 3 } & Mayor satisf. & Menor satisf. \\
\hline MN1: Dirección general & 8,69 & 5,10 \\
\hline MN2: Habilidades de marketing & 8,33 & 6,10 \\
\hline MN3: Conocimiento de idiomas & 8,93 & 6,23 \\
\hline MN4: Conocimiento de Informática y Nuevas Tecnologías & 8,69 & 8,05 \\
\hline MN5: Experiencia en el sector & 8,93 & 6,49 \\
\hline MN6: Experiencia en mercados internacionales & 8,45 & 27,92 \\
\hline FC2: N. empleados & 247,50 & 27,27 \\
\hline FC4: N. años exportando & 28,21 & 6,75 \\
\hline MS7: Relación con miembros del canal (agentes, distribuidores...) & 8,21 & 6,49 \\
\hline MS8: Imagen corporativa (pol. ambientales, éticas, sociales) & 8,57 & 7,27 \\
\hline MS9: Habilidad para retener a los clientes & 7,62 & 5,81 \\
\hline MS12: Incorporación de las NNTT en las políticas de marketing & & \\
\hline
\end{tabular}

Fuente: Elaboración propia. 
Los resultados muestran que, tanto unos mayores niveles de formación de los directivos, como una mayor experiencia en el mercado, se relacionan con un grado superior de satisfacción general en lo que a internacionalización se refiere. Del mismo modo, guardan una relación positiva otros activos intangibles de la empresa, en particular de la estrategia de marketing, como son los aspectos relacionales y el uso de las nuevas tecnologías. Y finalmente, otras características de la empresa (fundamentalmente tamaño y años de experiencia en mercados internacionales), guardan también una relación positiva con la rentabilidad empresarial. Estas conexiones, por tanto, contribuyen a la confirmación de las hipótesis planteadas y, como se ha demostrado en apartados anteriores, han sido defendidas también por diferentes autores (Chen et al., 2016; Parnell et al., 2015; Fernández \& Alegre, 2015; Prasad et al., 2001).

\section{DISCUSIÓN Y CONCLUSIONES}

El estudio que hemos llevado a cabo pretende analizar las características de las empresas exportadoras en el sector del calzado español mediante las principales variables que determinan el resultado exportador, con el fin de definir las fortalezas y debilidades de las empresas que operan en él. Contribuye, por tanto, al análisis del sector desde un punto de vista académico, utilizando como herramienta el análisis estadístico descriptivo.

Partimos del hecho de que esta industria presenta unas características muy peculiares, con empresas de tamaño medio (pymes en su mayoría), carácter y propiedad familiar, una considerable fragmentación, y una concentración geográfica en torno al levante español (provincia de Alicante fundamentalmente), sin el menosprecio de otras zonas productoras en La Rioja, Baleares, Cataluña o Andalucía.

Con respecto a la gestión, cabe destacar la importancia de las políticas de marketing, debido a la comercialización de un tipo de producto con un alto valor añadido basado en el diseño y la moda, operando en un entorno altamente cambiante y competitivo.

Las empresas seleccionadas, representativas del sector, muestran una cierta madurez, si bien comenzaron, en términos generales, su aventura empresarial en el mercado doméstico antes de considerar su presencia en mercados internacionales. Por otra parte, cuando lo hicieron optaron por los mercados más cercanos (bien en términos de distancia física o psíquica), que presentan mayores similitudes en cuanto a gustos y comportamientos del consumidor y del entorno. Operando dentro del mercado europeo evitan también la presencia de barreras arancelarias que pudieran mermar la competitividad empresarial y, en ocasiones, la existencia de otras barreras y/o trabas a la importación. De esta forma se demuestra la importancia del entorno internacional y de las características de la empresa en la estrategia empresarial, dos variables que han sido definidas y medidas en nuestra investigación.

La situación del sector podría, desde el punto de vista académico, identificarse con los modelos gradualistas de la escuela nórdica, cuyos máximos exponentes fueron Johanson y Vahlne en la década de los 70. Si bien el proceso gradual de internacionalización supone un proceso de aprendizaje, de forma que las experiencias del mercado doméstico permiten replicar un modelo similar en entornos exteriores parecidos y cercanos para posteriormente conquistar mercados más complejos, el sector debería considerar la opción de incrementar su presencia en mercados de alto valor añadido fuera de la Unión Europea, como pueden ser Rusia, Estados Unidos o países del sudeste asiático. Aunque algunas empresas han optado ya por esta estrategia, muchas otras no han dado ese paso todavía. El sector, por tanto, presenta la estructura de un mercado tradicional y maduro que, al contrario de empresas que nacen globales desde un principio (born globals), han hecho un gran esfuerzo por crecer en otros mercados internacionales.

Otro aspecto determinante en la consecución del resultado empresarial va a ser la gestión directiva de la empresa (Desarbo et al., 2005; Song et al., 2008; Murray et al., 2011; Morgan \& Katsikeas, 2012; Parnell et al., 2015). Su función principal será la de analizar los cambios constantes en el entorno (analizados en la literatura desde el punto de vista de las capacidades dinámicas y la teoría de la contingencia) y en el comportamiento de los consumidores para adaptar la estrategia de marketing internacional, uno de los principales activos intangibles de la empresa, ampliamente descritos por la teoría del punto de vista de los recursos 
(Resource Based View-RBV). Si bien en el caso del sector calzado, la formación de los directivos en marketing e internacionalización podría considerarse como adecuada, cuestiones como la gestión de equipos o la comunicación interna presentan todavía algunos síntomas de posible mejora. Además, esta investigación demuestra, gracias al análisis descriptivo de los datos recogidos, que unos mayores niveles de formación de los directivos repercuten en un mayor nivel de satisfacción con el resultado empresarial, lo cual apunta en la dirección de la literatura considerada. En consecuencia, una mayor formación y experiencia permiten un análisis más acertado de las condiciones que ejercen una influencia sobre la empresa, con el fin de definir, de forma mucho más eficiente, la estrategia competitiva de la misma.

Cuando hablamos del entorno, observamos que presenta una cierta relación con las características de los directivos de la empresa. Así pues, las condiciones de los mercados internacionales tendrán que ser interpretadas por ellos con el fin de adaptarse a las nuevas circunstancias. Además, las características de estos mercados van a determinar las habilidades del equipo de dirección. Esto queda reflejado en las escalas que hemos introducido, al valorar aspectos como la experiencia en mercados internacionales, la orientación a los mercados exteriores, o la formación en idiomas y marketing. Tal y como demuestra la literatura, la forma en la que los directivos adaptan su formación y la estrategia de marketing internacional, supondrá un mejor o peor resultado empresarial (Fernández \& Alegre, 2015; Contrator et al., 2005; Lages \& Montgomery, 2005; Stöttinger \& Holzmüller, 2001). Las mismas conclusiones se desprenden también del análisis descriptivo de esta investigación.

En lo que respecta a la implantación de la estrategia de marketing internacional, es evidente que se ha hecho un gran esfuerzo por incorporar matices a la relación tradicional con el cliente para conseguir su fidelización a largo plazo, en consonancia con la literatura (Kotler, 2010; Kotler et al., 2016, 2021; Chugan \& Singh, 2014; Payne \& Frow, 2017). También se han introducido aspectos de mejora en la imagen corporativa mediante acciones de responsabilidad social corporativa que pretenden involucrar al consumidor en la filosofía de la empresa (Orlitzky et al., 2011; Chen et al., 2016). Los datos analizados muestran, por tanto, que las empresas de la muestra están haciendo un esfuerzo considerable por avanzar en la dirección que apuntan los principales estudios académicos. Queda, sin embargo, bastante camino por recorrer en torno a la incorporación de las nuevas tecnologías a las estrategias de marketing, fundamentalmente en aspectos como el marketing digital, la venta a través de internet, o el tratamiento de la información obtenida de los propios consumidores, que han sido claramente vinculados con un mayor rendimiento empresarial en lo que a actividad internacional se refiere (Parnell et al., 2015). No obstante, lo que es evidente, es que las políticas de marketing internacional en el sector del calzado están avanzando desde una concepción basada en los productos a otra basada en el mercado y los servicios, considerándose al cliente como el punto de partida de todas las estrategias, tal y como apuntan diversos autores (Vargo \& Lusch, 2004; 2016).

Finalmente, diremos que nuestro estudio se centra en el análisis de dos aspectos fundamentales en lo que a recursos empresariales intangibles se refiere: el conocimiento (considerado en el análisis de la función directiva) y la estrategia de marketing internacional. Quedan, por tanto, para futuras investigaciones, otros aspectos (como la incorporación de la tecnología a los procesos y estrategias empresariales) que deberían ser considerados en mayor profundidad. En este sentido, el uso de Internet en los procesos de internacionalización y su análisis desde el punto de vista de los recursos es un tema que, si bien ha recibido importantes contribuciones (Prasad et al., 2001; Morgan \& Katsikeas, 2012; Parnell et al., 2015), debe presentar todavía importantes avances en el futuro más inmediato.

Comentar también, en lo que a limitaciones se refiere, que el tamaño de la muestra no es excesivamente elevado. No obstante, la selección de empresas consideradas en la misma (que se consideran representativas del sector debido al amplio conocimiento del mismo), viene a suplir en parte esas limitaciones. Una selección alternativa podría presentar resultados ligeramente diferentes, si bien las conclusiones no sufrirían grandes alteraciones.

\section{Declaración responsable y conflicto de intereses}

El autor declara que no existe ningún conflicto de interés con relación a la publicación de este artículo. 


\section{REFERENCIAS}

Aaby, N. E. \& Slater, S. F. (1989). Management influences on export performance: a review of the empirical literature 1978-88. International marketing review, 6(4), 7-26.https://doi.org/10.1108/EUM0000000001516

Alvarez, R. (2004). Sources of export success in small-and medium-sized enterprises: the impact of public programs. International Business Review, 13(3), 383-400.https://doi.org/10.1016/j.ibusrev.2004.01.002

Baldauf, A., Cravens, D. W.\& Wagner, U. (2000). Examining determinants of export performance in small open economies. Journal of World Business, 35(1), 61-79.https://doi.org/10.1016/S1090-9516(99)00034-6

Barney, J. (1991). Firm resources and sustained competitive advantage. Journal of management, 17(1), 99-120. https://doi. org/10.1177/014920639101700108

Bilkey, W. J. (1978). An attempted integration of the literature on the export behavior of firms. Journal of international Business studies, 9(1), 33-46. https://doi.org/10.1057/palgrave.jibs.8490649

Bouncken, R. B., Schuessler, F. \& Kraus, S. (2015). The theoretical embedding of born globals: Challenging existing internationalization theories. International Business \& Economics Research Journal (IBER), 14(1), 39-46. https://doi. org/10.19030/iber.v14i1.9030

Cavusgil, S. T. \& Zou, S. (1994). Marketing strategy-performance relationship: an investigation of the empirical link in export market ventures. Journal of marketing, 58(1), 1-21. https://doi.org/10.1177/002224299405800101

CESCE, Compañía Española de Seguros de Crédito a la Exportación (2018). Informe sectorial de la economía española. https://issuu.com/cesce.es/docs/informe_sectorial_2018

CESCE, Compañía Española de Seguros de Crédito a la Exportación (2019). Informe sectorial de la economía española. https://issuu.com/cesce.es/docs/informe_sectorial_cesce_2019

Chabowski, B., Kekec, P., Morgan, N. A., Hult, G. T. M., Walkowiak, T. \& Runnalls, B. (2018). An assessment of the exporting literature: Using theory and data to identify future research directions. Journal of International Marketing, 26(1), 118143. https://doi.org/10.1509/jim.16.0129

Chen, J., Sousa, C. M. \& He, X. (2016). The determinants of export performance: a review of the literature 2006-2014. International Marketing Review. https://doi.org/10.1108/IMR-10-2015-0212

Chugan, P. K. \& Singh, S. (2014). Taxonomy for firm-level determinants of export performance. Universal Journal of Industrial and Business Management, 2(1), 6-12. https://doi.org/10.13189/ujibm.2014.020102

Contractor, F. J., Hsu, C. C. \& Kundu, S. K. (2005). Explaining export performance: a comparative study of international new ventures in Indian and Taiwanese software industry. MIR: Management International Review, 83-110.

DeSarbo, W. S., Anthony Di Benedetto, C., Song, M. \& Sinha, I. (2005). Revisiting the Miles and Snow strategic framework: uncovering interrelationships between strategic types, capabilities, environmental uncertainty, and firm performance. Strategic management journal, 26(1), 47-74. https://doi.org/10.1002/smj.431

Fernández-Mesa, A. \& Alegre, J. (2015). Entrepreneurial orientation and export intensity: Examining the interplay of organizational learning and innovation. International Business Review, 24(1), 148-156. https://doi.org/10.1016/j.ibusrev.2014.07.004

Francis, J. \& Collins-Dodd, C. (2004). Impact of export promotion programs on firm competencies, strategies and performance. International Marketing Review. https://doi.org/10.1108/02651330410547153

Grant, R. M. (1996). Prospering in dynamically-competitive environments: Organizational capability as knowledge integration. Organization science, 7(4), 375-387. https://doi.org/10.1287/orsc.7.4.375

Green, K. M., Covin, J. G. \& Slevin, D. P. (2008). Exploring the relationship between strategic reactiveness and entrepreneurial orientation: The role of structure-style fit. Journal of Business Venturing, 23(3), 356-383. https://doi.org/10.1016/j.jbusvent.2007.01.002

Gummesson, E. (2002). Relationship marketing and a new economy: it's time for de-programming. Journal of services marketing. https://doi.org/10.1108/08876040210447315

ICEX - Instituto Español de Comercio Exterior (2021). Base de datos ESTACOM. https://www.icex.es/icex/es/navegacion-principal/todos-nuestros-servicios/informacion-de-mercados/estadisticas/sus-estadisticas-a-medida/estadisticas-espanolas-estacom/index.html

IVACE - Instituto Valenciano de Competitividad Empresarial (2019). Análisis del sector calzado. https://www.ivace.es/Internacional_Informes-Publicaciones/Sectores-enlaces/CALZADO.pdf 
Katsikeas, C. S., Leonidou, L. C. \& Morgan, N. A. (2000). Firm-level export performance assessment: review, evaluation, and development.Journal of the Academy of Marketing Science, 28(4), 493-511. https://doi.org/10.1177/0092070300284003

Katsikeas, C. S., Samiee, S. \& Theodosiou, M. (2006). Strategy fit and performance consequences of international marketing standardization. Strategic management journal, 27(9), 867-890. https://doi.org/10.1002/smj.549

Kotler, P. (2010). Marketing 3.0: from products to customers to the human spirit (No. 658.8 K8487m Ej. 1). JOHN WILLEY \& SONS. https://doi.org/10.1002/9781118257883

Kotler, P., Kartajaya, H. \& Setiawan, I. (2016). Marketing 4.0: Moving from traditional to digital. John Wiley \& Sons.

Kotler, P., Kartajaya, H. \& Setiawan, I. (2021). Marketing 5.0: Technology for Humanity. John Wiley \& Sons.

Lages, L. F. \& Montgomery, D. B. (2005). The relationship between export assistance and performance improvement in Portuguese export ventures. European Journal of marketing.

Leonidou, L. C., Katsikeas, C. S. \& Samiee, S. (2002). Marketing strategy determinants of export performance: a meta-analysis. Journal of Business research, 55(1), 51-67. https://doi.org/10.1016/S0148-2963(00)00133-8

Lipuma, J. A., Newbert, S. L. \& Doh, J. P. (2013). The effect of institutional quality on firm export performance in emerging economies: a contingency model of firm age and size. Small Business Economics, 40(4), 817-841. https://doi. org/10.1007/s11187-011-9395-7

Morgan, R. M. \& Hunt, S. D. (1994). The commitment-trust theory of relationship marketing. Journal of marketing, 58(3), 20-38. https://doi.org/10.2307/1252308

Morgan, N. A., Katsikeas, C. S. \& Vorhies, D. W. (2012). Export marketing strategy implementation, export marketing capabilities, and export venture performance. Journal of the academy of marketing science, 40(2), 271-289. https:// doi.org/10.1007/s11747-011-0275-0

Murray, J. Y., Gao, G. Y. \& Kotabe, M. (2011). Market orientation and performance of export ventures: the process through marketing capabilities and competitive advantages. Journal of the Academy of Marketing Science, 39(2), $252-269$.

https://doi.org/10.1007/s11747-010-0195-4

Nelson, R. R. (1991). Why do firms differ, and how does it matter? Strategic management journal, 12(S2), 61-74. https://doi. org/10.1002/smj.4250121006

O'Cass, A. \& Julian, C. (2003). Examining firm and environmental influences on export marketing mix strategy and export performance of Australian exporters. European journal of marketing. https://doi.org/10.1108/03090560310459005

Orlitzky, M., Siegel, D. S. \& Waldman, D. A. (2011). Strategic corporate social responsibility and environmental sustainability. Business \& society, 50(1), 6-27. https://doi.org/10.1177/0007650310394323

Parnell, J. A., Long, Z., \& Lester, D. (2015). Competitive strategy, capabilities and uncertainty in small and medium sized enterprises (SMEs) in China and the United States. Management Decision. https://doi.org/10.1108/MD-04-2014-0222

Payne, A. \& Frow, P. (2005). A strategic framework for customer relationship management. Journal of marketing, 69(4), 167-176. https://doi.org/10.1509/jmkg.2005.69.4.167

Payne, A. F., Storbacka, K. \& Frow, P. (2008). Managing the co-creation of value. Journal of the academy of marketing science, 36(1), 83-96. https://doi.org/10.1007/s11747-007-0070-0

Payne, A. \& Frow, P. (2017). Relationship marketing: looking backwards towards the future. Journal of services marketing. https://doi.org/10.1108/JSM-11-2016-0380

Prahalad, C. K. \& Hamel, G. (1990). Core competency concept. Harvard Business Review, 64(3), 70-92.

Prasad, V. K., Ramamurthy, K. \& Naidu, G. M. (2001). The influence of Internet-marketing integration on marketing competencies and export performance. Journal of International Marketing, 9(4), 82-110. https://doi.org/10.1509/jimk.9.4.82.19944

Ruzzier, M., Hisrich, R. D. \& Antoncic, B. (2006). SME internationalization research: past, present, and future. Journal of Small Business and Enterprise Development, 13(4), 476-497. https://doi.org/10.1108/14626000610705705

Song, M., Nason, R. W. \& Di Benedetto, C. A. (2008). Distinctive marketing and information technology capabilities and strategic types: A cross-national investigation. Journal of International Marketing, 16(1), 4-38. https://doi.org/10.1509/jimk.16.1.4

Sousa, C. M. (2004). Export performance measurement: an evaluation of the empirical research in the literature. Academy of marketing science review, 2004, 1.

Sousa, C. M., Martínez-López, F. J. \& Coelho, F. (2008). The determinants of export performance: A review of the research in the literature between 1998 and 2005. International Journal of Management Reviews, 10(4), 343-374. https://doi. org/10.1111/j.1468-2370.2008.00232.x 
Sousa, C. M. \& Lengler, J. (2009). Psychic distance, marketing strategy and performance in export ventures of Brazilian firms. Journal of Marketing Management, 25(5-6), 591-610. https://doi.org/10.1362/026725709X461876

Stöttinger, B. \& Holzmüller, H. H. (2001). Cross-national stability of an export performance model-a comparative study of Austria and the US. MIR: Management International Review, 7-28.

Teece, D. J., Pisano, G. \& Shuen, A. (1997). Dynamic capabilities and strategic management. Strategic management journal, 18(7), 509-533. https://doi.org/10.1002/(SICI)1097-0266(199708)18:7<509::AID-SMJ882>3.0.CO;2-Z

Vargo, S. L. \& Lusch, R. F. (2004). Evolving to a new dominant logic for marketing. Journal of marketing, 68(1), 1-17. https:// doi.org/10.1509/jmkg.68.1.1.24036

Vargo, S. L. \& Lusch, R. F. (2016). Institutions and axioms: an extension and update of service-dominant logic. Journal of the Academy of marketing Science, 44(1), 5-23. https://doi.org/10.1007/s11747-015-0456-3

Wernerfelt, B. (1984). A resource-based view of the firm. Strategic management journal, 5(2), 171-180. https://doi. org/10.1002/smj.4250050207

Wernerfelt, B. (1995). The resource-based view of the firm: Ten years after. Strategic management journal, 16(3), $171-174$. https://doi.org/10.1002/smj.4250160303

White, D. S., Griffith, D. A. \& Ryans, J. K. (1998). Measuring export performance in service industries. International Marketing Review.

Zhu, Z., Zhao, J. \& Jin, X. (2013). A typology of net-enabled organisational capabilities for digital competitive advantage: the case study of travel and hospitality industry in China. International Journal of Networking and Virtual Organisations 11, 12(1), 56-69.

https://doi.org/10.1504/IJNVO.2013.051751

Zou, S. \& Stan, S. (1998). The determinants of export performance: a review of the empirical literature between 1987 and 1997. International marketing review. https://doi.org/10.1108/02651339810236290 


\section{ANEXO I}

\section{DETALLE DEL CUESTIONARIO ENVIADO A LAS EMPRESAS DE LA MUESTRA}

INFORMACIÓN GENERAL DE LA EMPRESA

\begin{tabular}{|l|l|}
\hline Razón social & \\
\hline Ciudad & \\
\hline Provincia / Comunidad Autónoma & \\
\hline Facturación total (€) & \\
\hline N. empleados & \\
\hline N. años funcionamiento & \\
\hline N. años exportando & \\
\hline N. países a los que exporta & \\
\hline Intensidad exportadora: cifra exportación/total de ventas (\%) & \\
\hline Exportaciones Unión Europea / Total exportaciones (\%) & \\
\hline
\end{tabular}

\section{CARACTERÍSTICAS DE LOS DIRECTIVOS (MANAGEMENT)}

Por favor, evalúe el desempeño de su empresa en lo que respecta a las características de la dirección, en comparación con su competencia más directa:

\begin{tabular}{|l|c|c|c|c|c|c|c|}
\multicolumn{1}{|c|}{ mucho peor } & \multicolumn{7}{c|}{ mucho mejor } \\
\hline Dirección general & 1 & 2 & 3 & 4 & 5 & 6 & 7 \\
\hline Habilidades de marketing & 1 & 2 & 3 & 4 & 5 & 6 & 7 \\
\hline Conocimiento de idiomas & 1 & 2 & 3 & 4 & 5 & 6 & 7 \\
\hline Conocimiento de Informática y Nuevas Tecnologías & 1 & 2 & 3 & 4 & 5 & 6 & 7 \\
\hline Experiencia en el sector & 1 & 2 & 3 & 4 & 5 & 6 & 7 \\
\hline Experiencia en mercados internacionales & 1 & 2 & 3 & 4 & 5 & 6 & 7 \\
\hline Visión general de la empresa & 1 & 2 & 3 & 4 & 5 & 6 & 7 \\
\hline Orientación a la exportación & 1 & 2 & 3 & 4 & 5 & 6 & 7 \\
\hline Habilidades para facilitar la comunicación en la empresa & 1 & 2 & 3 & 4 & 5 & 6 & 7 \\
\hline Gestión de equipos & 1 & 2 & 3 & 4 & 5 & 6 & 7 \\
\hline
\end{tabular}




\section{ESTRATEGIA DE MARKETING}

Por favor, evalúe el desempeño de su empresa en lo que respecta a los siguientes componentes de la estrategia de marketing, en comparación con su competencia más directa:

\begin{tabular}{|c|c|c|c|c|c|c|c|}
\hline \multicolumn{6}{|c|}{ Mucho peor } & \multicolumn{2}{|c|}{ mucho mejo } \\
\hline Conocimiento de los clientes & 1 & 2 & 3 & 4 & 5 & 6 & 7 \\
\hline Conocimiento de la competencia & 1 & 2 & 3 & 4 & 5 & 6 & 7 \\
\hline Planificación de las actividades de marketing & 1 & 2 & 3 & 4 & 5 & 6 & 7 \\
\hline Integración actividades de marketing & 1 & 2 & 3 & 4 & 5 & 6 & 7 \\
\hline Habilidad para segmentar y reconocer mercados & 1 & 2 & 3 & 4 & 5 & 6 & 7 \\
\hline Desarrollo de proyectos de investigación de mercados & 1 & 2 & 3 & 4 & 5 & 6 & 7 \\
\hline Relación con los miembros del canal (agentes, distribuidores...) & 1 & 2 & 3 & 4 & 5 & 6 & 7 \\
\hline Imagen corporativa (políticas ambientales, éticas, sociales) & 1 & 2 & 3 & 4 & 5 & 6 & 7 \\
\hline Habilidad para retener a los clientes & 1 & 2 & 3 & 4 & 5 & 6 & 7 \\
\hline Efectividad del servicio post-venta & 1 & 2 & 3 & 4 & 5 & 6 & 7 \\
\hline Efectividad del equipo comercial & 1 & 2 & 3 & 4 & 5 & 6 & 7 \\
\hline Incorporación de las nuevas tecnologías en las políticas de mktg. & 1 & 2 & 3 & 4 & 5 & 6 & 7 \\
\hline
\end{tabular}

\section{ENTORNO}

Por favor, evalúe la importancia de los siguientes aspectos a la hora de determinar en qué mercados internacionales operar:

\begin{tabular}{|l|l|l|l|l|l|l|}
\multicolumn{4}{|c|}{ poca importancia } & \multicolumn{4}{c|}{ mucha importancia } \\
\hline 1 & 2 & 3 & 4 & 5 & 6 & 7 \\
\hline 1 & 2 & 3 & 4 & 5 & 6 & 7 \\
\hline 1 & 2 & 3 & 4 & 5 & 6 & 7 \\
\hline 1 & 2 & 3 & 4 & 5 & 6 & 7 \\
\hline 1 & 2 & 3 & 4 & 5 & 6 & 7 \\
\hline 1 & 2 & 3 & 4 & 5 & 6 & 7 \\
\hline 1 & 2 & 3 & 4 & 5 & 6 & 7 \\
\hline 1 & 2 & 3 & 4 & 5 & 6 & 7 \\
\hline 1 & 2 & 3 & 4 & 5 & 6 & 7 \\
\hline
\end{tabular}




\section{RESULTADO EXPORTADOR DE LA EMPRESA}

Por favor, determine el grado de satisfacción con la actividad internacional de su empresa en los últimos 3 años:

\begin{tabular}{|c|c|c|c|c|c|c|c|}
\hline \multicolumn{6}{|c|}{ Muy insatisfecho } & \multicolumn{2}{|c|}{ muy satisfecho } \\
\hline Volumen de exportaciones & 1 & 2 & 3 & 4 & 5 & 6 & 7 \\
\hline Crecimiento de las exportaciones & 1 & 2 & 3 & 4 & 5 & 6 & 7 \\
\hline Cuota de mercado (exportación) & 1 & 2 & 3 & 4 & 5 & 6 & 7 \\
\hline Grado de diversificación ( $n^{\circ}$ de países a los que exporta) & 1 & 2 & 3 & 4 & 5 & 6 & 7 \\
\hline Acceso a los mercados internacionales & 1 & 2 & 3 & 4 & 5 & 6 & 7 \\
\hline Rentabilidad de las exportaciones & 1 & 2 & 3 & 4 & 5 & 6 & 7 \\
\hline Margen comercial de las exportaciones & 1 & 2 & 3 & 4 & 5 & 6 & 7 \\
\hline Satisfacción general con la exportación & 1 & 2 & 3 & 4 & 5 & 6 & 7 \\
\hline
\end{tabular}

